\title{
THE INFLUENCE OF QUANTIZATION PROCESS ON THE PERFORMANCE OF GLOBAL ENTROPIC THRESHOLDING ALGORITHMS USING ELECTRICAL CAPACITANCE TOMOGRAPHY DATA
}

\author{
AJ Mwambela \\ Department of Physics, University of Dar es Salaam, \\ P.O. Box 35063, Dar es Salaam, Tanzania. \\ E-mail: nkomo98@yahoo.com
}

\begin{abstract}
In measuring component fraction in multiphase flows using tomographic techniques, it is desirable to use a high speed tomography system capable of generating 100 tomograms per second. The electrical capacitance tomography system in this regard is considered to be the best among the available tomographic techniques. However, due to its inherent limitations the system generates distorted reconstructed tomograms necessitating the use of extra signal processing techniques such as thresholding to minimize these distortions. Whilst thresholding technique has been effective in minimizing distortions, the additional computation associated with the process limits the speed of tomogram generation desired from the system. Further, the accuracy of the techniques is limited to higher ranges of the full component fraction range. However, since its performance can be influenced by the nature of the quantization process required a priori, optimal quantization parameters can be found and used to improve performance. In this article the influence of quantization resolution and its rate on the performance of global entropic thresholding algorithms have been investigated. Measurement of gas volume component fraction in a multiphase flow of gas/liquid mixture using electrical capacitance tomography system has been used for evaluation using simulated and online capacitance measurement data. Results show that an optimal quantizer resolution is flow regime dependent. Higher resolutions are optimal for annular flow and vice versa for stratified flow regimes. Also, higher resolution significantly minimizes the dependency of the thresholding algorithm on the object to be searched, thereby reducing complexity of designing a thresholder. Overall, the optimal quantization resolution is 256.
\end{abstract}

\section{INTRODUCTION}

In measuring component fraction in multiphase flows using tomographic techniques, it is desirable to use a high speed tomography system capable of generating 100 or above tomograms per second. This allows capture of real-time data of the turbulent fluctuation in the flow field. The Electrical Capacitance Tomography (ECT) system in this regard is considered to be the best among the available tomographic techniques because of its high-speed capability. The ECT system has been developed to image industrial process containing dielectric material such as gas/oil flows in oil pipelines. It measures the changes in inter-electrode capacitance due to high permittivity material present in the low permittivity background, and reconstruct cross-sectional image from the measured data (Yang 1996, 2001). However, due to its inherent limitations the system generates distorted reconstructed tomograms. The most critical problems still facing the system are its relatively low spatial resolution and inaccurate reconstructed tomograms using a fast but crude Linear Back Projection (LBP) reconstruction algorithm (Isaksen 1996). In addressing the problem of inaccurate reconstructed tomograms, researchers have used various approaches to meet the desired requirements (Waritso \& Fan 2001). One of the approach investigated by the author is the use of 
signal processing techniques namely thresholding and data fusion to improve the accuracy of information extracted from the reconstructed tomograms (Mwambela \& Johansen 2001, Mwambela 1999, Mwambela et al. 1997). Whilst the combination of thresholding and fusion techniques have been show to be effective in minimizing distortions, the additional computation associated with the process limit the speed of tomogram generation desired from the system. Further, the accuracy of the techniques is limited to higher ranges of the full component fraction range.

However, since the performance of thresholding techniques can be influenced by the nature of the quantization process required a priori, optimal quantization parameters can be found and used to improve overall performance. Quantization process converts analogue tomogram data into digital tomogram data suitable for use by thresholding algorithms. A quantizer is completely described by its output levels and the corresponding partitioning cells. The principle goal of quantizer design is to select the output levels and partition cells so as to provide the minimum possible average distortion for a fixed number of allowable output levels or, equivalently, a fixed resolution. Large values of allowable output levels indicate the accuracy with which the original input data is described. At the current state of the art of computer technology, the most common numbers of allowable output levels used in quantization process are 16, 64, 256 and 512 for industrial applications, although the number may go up to 1024 levels for medical applications (Galbiati 1990). On the other hand large values of allowable output make thresholding process computationally intensive affecting the speed of tomogram generation. It can be seen that the desirable accuracy of the quantizer and the speed of tomogram generation objectives are conflicting and a compromise is needed. To find the best trade-off it is first necessary to understand what is the best that can be achieved under the given constraints.

This article reports the influence the quantization resolution can have on the performance of entropic thresholding algorithms. The main objective was to find an optimal quantization resolution and thresholding parameters, which gives the best compromise between the speed of reconstruction and the accuracy of the reconstructed tomograms for annular flow regime over the full component fraction range.

Global entropic thresholding algorithm of Kapur et al. (1985) has been used. Unsupervised thresholding approach where the best thresholding grey level is selected by exhaustive search among available grey levels has been implemented. The scalar fixed length uniform quantizer has been implemented and 16, 256 and 512 quantizer resolutions have been used in the evaluation experiment. Measurement of gas volume component fraction in a multiphase flow of gas/liquid mixture using electrical capacitance tomography system has been used for evaluation using computer simulated and online capacitance measurement data.

\section{MATERIAL AND METHODS The Measurement System Theory}

The ECT system has been developed to image industrial process containing dielectric material such as gas/oil flows in oil pipelines. It measures the changes in inter-electrode capacitance due to high permittivity material present in the low permittivity background, and reconstructs cross-sectional tomogram (image) from the measured data (Yang 1996, 2001). From signal processing point of view the ECT system can be seen as composed of the sampler, quantizer and thresholder subsystems (Figure 1). Tomograms generated from this system basically are two-dimensional functions of space; i.e. the 
signal $f(x, y)$ is a mathematical representation of a tomogram, where $f(x, y)$ is the brightness value at spatial co-ordinate $(x, y)$. As a result, tomograms can be classified by whether or not they are defined over all points in the spatial domain and by whether their brightness values are represented with finite or infinite precision (Table 1). As it turns out, different categories of tomograms appears at different stages of the measurement system. The analysis that follows is based on the nature of the tomograms generated at different stages according to the described categorization.

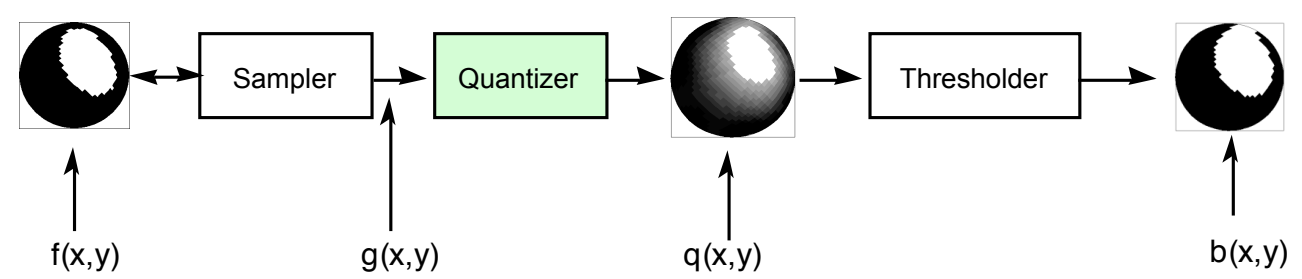

Figure 1: Block diagram showing the measurement system used in this work

Table 1: $\quad$ Categorization of tomograms.

\begin{tabular}{llc}
\hline \multicolumn{1}{c}{ Tomogram Category } & \multicolumn{1}{c}{ Description } & Examples \\
\hline Continuous-continuous & $\begin{array}{l}\text { A tomogram that is defined at all set of points and its } \\
\text { tomogram values have infinite precision. }\end{array}$ & $f(x, y)$ \\
Discrete-continuous & $\begin{array}{l}\text { A tomogram that is defined only at discrete set of points } \\
\text { and its tomogram values have infinite precision. }\end{array}$ & $g(x, y)$ \\
Discrete-discrete & $\begin{array}{l}\text { A tomogram that is defined only at discrete set of points } \\
\text { and its tomogram values have finite precision. }\end{array}$ & $q(x, y) b(x, y)$ \\
\hline
\end{tabular}

\section{The Sampler}

The ECT system, together with its associated LBP reconstruction algorithm, constitutes the sampler whose main function is to convert continuous-continuous tomogram $f(x, y)$ into the discretecontinuous form $g(x, y)$. The implemented LBP reconstruction algorithm (Xie et al., 1992) is described by the following equation.

$$
g(x, y)=\left(\sum_{i=1}^{m-1} \sum_{j=i+1}^{m} S_{i j} N_{i j}\right) \times\left(\sum_{i=1}^{m-1} \sum_{j=i+1}^{m} S_{i j}\right)^{-1}
$$

where $m$ is the number of electrodes used, $S_{i j}$ is the sensitivity distribution and $N_{i j}$ the normalized capacitance, both for electrode pair $i j$.

\section{The Quantizer}

The function of a quantizer is to convert discrete-continuous tomogram $g(x, y)$ from the sampler into discrete-discrete form $q(x$, y). In its simplest form, a quantizer observes a single brightness value and select the nearest approximating values from a 
predetermined finite set of allowed numerical values. More precisely, a quantizer $Q$ is defined as a mapping from source alphabet $R^{K}$ to a reproduction codebook $C=\left\{\hat{x}_{i}\right\}_{i \in I} \subset R^{K}$, where $I$ is an arbitrary countable index set. In fact, a quantizer can be viewed as a combination effect of two successive mappings, an encoder $(\alpha)$ and a decoder $(\beta)$ such that $Q=\beta$ o $\alpha$. The lossy encoder $\alpha: R^{K} \rightarrow I$ is specified by a partition of $R^{K}$ into $\mathrm{p}$ a r t i t i o n e $11 \mathrm{~s}$ $S_{i}=\left\{x \in R^{K} \mid \alpha(x)=i\right\} i \in I . \quad$ T h e reproduction decoder $\beta: I \rightarrow R^{K} \quad$ is specified by the code-book $\mathrm{C}$. If $\mathrm{K}=1$, the quantizer is a scalar quantizer, otherwise it is a vector quantizer.

A uniform quantizer is a regular quantizer in which a) the boundary points are equally spaced, b) the output levels for granular cells are the mid-point of the quantization intervals. Truncation and rounding operations in approximating real numbers are examples of uniform quantizer. The quality of a quantizer is determined by its distortion and rate. Distortion measure $d(x, \hat{x})$ quantifies the goodness of the resulting reproduction in comparison to the original. The most common measure of distortion is the Mean Square Error (MSE) and the Absolute Error (AE) defined by eqns. (2) and (3) respectively.

$$
\begin{gathered}
d(x, \hat{x})_{M S E}=\|x-\hat{x}\|^{2}=\frac{1}{N} \sum_{i=1}^{N}\left(x_{i}-\hat{x}_{i}\right)^{2} \\
d(x, \hat{x})_{A E}=\|x-\hat{x}\|=\frac{1}{N} \sum_{i=1}^{N}\left(x_{i}-\hat{x}_{i}\right)
\end{gathered}
$$

where $x$ is the input sample and $\hat{x}=Q(x)$ is the reproduce output value. The absolute error is sometimes referred to as Distribution Error (DE) in process tomography community. It is desirable to have the average distortion as small as possible, and in fact negligible average distortion is achievable by letting the cells becomes numerous and tiny.

The resolution or code rate ( $r$ ) of a scalar quantizer is defined as $r=\log _{2} I$. The resolution measure the number of bits needed to uniquely specify the quantized values. It also indicates the accuracy with which the original signal is described. Specifically, if $\mathrm{r}$ is an integer, one would assign to each $\hat{x}_{i}$ a unique binary r-turple (by coding the value $\hat{x}_{i}$ into binary codewords in an invertible manner). A quantizer with fixed-length binary code-words is said to have a fixed rate because all quantizer levels are assumed to have binary codewords of equal length. A variable rate quantizer has variable-length binary codewords and different quantized values can be specified with binary code-words of different length. Comprehensive treatment of quantization process can be found somewhere else (Gray and Neuhoff 1998).

\section{The Thresholder}

In its simple form, the thresholder observes a single brightness value $g(x, y)$ and classifies it between the two extreme predefined finite values $\mathrm{b}(x, y)$. The criteria function upon which this classification is based varies depending on the technique. When the principle of maximum entropy is exploited in such a way that the entropy of the data is used to select the appropriate thresholding grey level, the technique is called entropic thresholding technique. One of the best entropic thresholding techniques 
tested (Mwambela 1999) is that of Kapur et al. (1985) described as follows.

Let $N$ be the total number of pixel elements in a tomogram, $n$ the total number of grey levels and $f_{i}$ the frequency distribution of the $i^{\text {th }}$ grey level such that $\sum_{i=1}^{n} f_{i}=N$ where $i \in[1, \mathrm{n}]$. The entropy of a

$$
H_{n}=-\sum_{i=1}^{s} p_{i} \ln p_{i}-\sum_{i=1+s}^{n} p_{i} \ln p_{i}
$$

tomogram, according to Shannon (Shannon and Weaver, 1947), is defined as

$$
H_{n}=-\sum_{i=1}^{n} p_{i} \cdot \ln p_{i}
$$

where $\quad p_{i}=\frac{f_{i}}{N}$ is the probability of occurrence of the $i^{\text {th }}$ grey level.

The a priori information of oil and gas tomogram $q(x, y)$ can be defined as

On the other hand, a thresholded tomogram $b(x, y)$ will have two grey levels, resulting in what is known as a Binary Symmetrical Source (BSS), a source which produces a sequence of independent symbols that assumes the value of $z_{o}$ with probability $p$ and $z_{l}$ with probability $(1-p)$. Therefore its a posteriori entropy $H_{n}^{\prime}$ can be defined as

$$
H_{n}^{\prime}=-P_{s} \ln P_{s}-\left(1-P_{s}\right) \ln \left(1-P_{s}\right)
$$

where

$$
P_{s}=\sum_{i=1}^{s} p_{i} \text { and }\left(1-P_{s}\right)=\sum_{i=s+1}^{n} p_{i} .
$$

In order to find a suitable thresholding grey level s, Kapur et al. (1985) maximizes the following equation,

$$
H T_{s}=-\sum_{i=1}^{s} \frac{p_{i}}{P_{s}} \ln \left(\frac{p_{i}}{P_{s}}\right)-\sum_{i=s+1}^{n} \frac{p_{i}}{\left(1-P_{s}\right)} \ln \left(\frac{p_{i}}{\left(1-P_{s}\right)}\right)
$$

used in this work, have been derived from the proposed measurement system configuration. Note that in the table the first half of the label indicates the name of the algorithm used at quantizer or thresholder stage. The first letter in the second half indicates the type of truncation mechanism used at the quantizer stage, and the last letter indicates the object searched by the thresholder, where applicable. The letter ' $A$ ' stands for sampled whereas the letter ' $Q$ ' stands for quantized. 
Mwambela. - The influence of quantization process on the perfomance ...

Table 2: List of algorithms used in this work.

\begin{tabular}{ll}
\hline \multicolumn{1}{c}{ Algorithms } & \multicolumn{1}{c}{ Description } \\
\hline LBP_SA & Conventional LBP algorithm \\
LBP_SQ & Quantized conventional LBP algorithm. \\
KAP_SG & $\begin{array}{l}\text { Unsupervised Kapur et al. thresholding algorithm with simple truncation } \\
\text { mechanism and gas as the object to be searched }\end{array}$ \\
KAP_SL & $\begin{array}{l}\text { Unsupervised Kapur et al. thresholding algorithm with simple truncation } \\
\text { mechanism and liquid as the object to be searched }\end{array}$ \\
\hline
\end{tabular}

\section{The Experiment}

Measurements of component fraction in a two multiphase flow of oil and gas were used in the evaluation process. Two types of data, computer simulated and on-line, were used. Computer simulated capacitance tomogram data were generated from an 8electrodes capacitance sensor system simulator (Isaksen 1994). Desired static annular flow regimes over the full component fraction range were obtained by selectively feeding parameters to the simulator such as radius of the air bubble, permittivity constants of materials involved etc which specified the flow regime to be imaged, then reconstruct the corresponding tomogram using the capacitance sensory simulator.

On-line capacitance tomogram data were obtained from the actual measurement of the gas/oil mixture flow using the ECT system taken from the Christian Michelsen Research (CMR) multiphase laboratory test rig shown in Figure 2 (Midttveit et al. 1997). However, it must be pointed out that the oil phase in this test rig contains a small amount of water at $1 \%$ water-cut due to difficulty in completely removing water in the liquid phase introduced when conducting other experiments.

The performances of the thresholding algorithms were evaluated based on the design of quantizer resolution. The focus of the evaluation process was limited to annular flow regimes only. This is because the flow rig used to generate on-line data can reasonably give annular flow regimes, which are similar to those obtained from the simulator. 


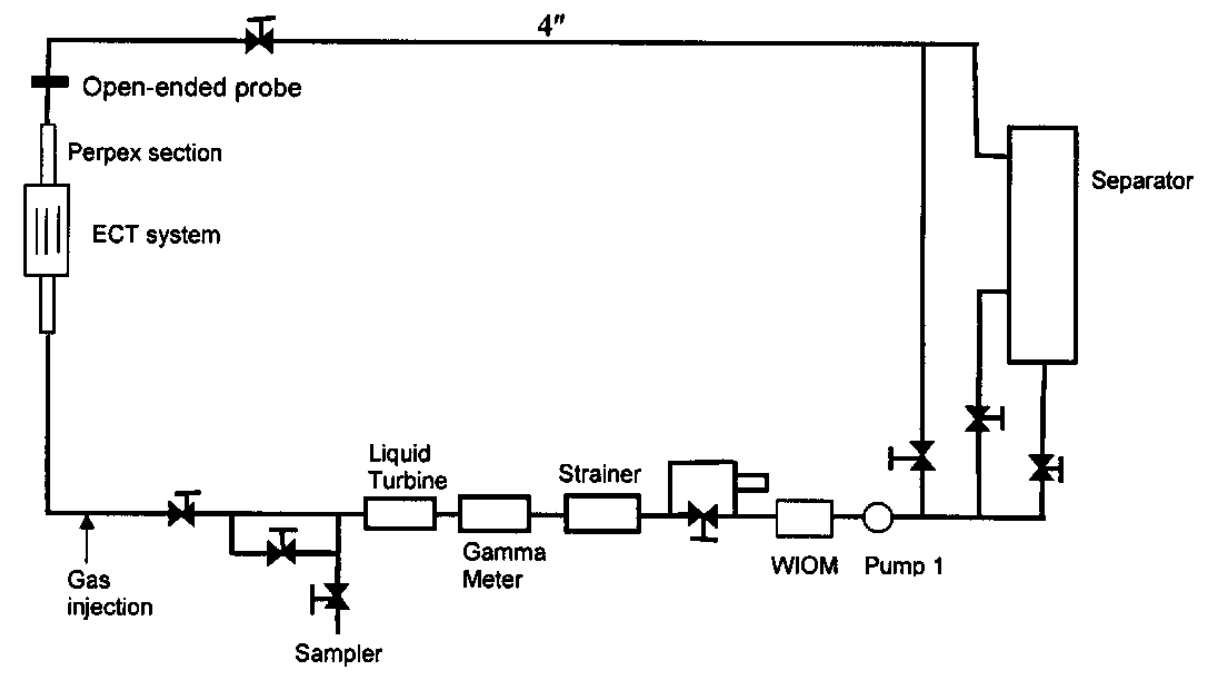

Figure 2: A sketch diagram of CMR laboratory multiphase flow test rig. The capacitance sensor unit is mounted on a vertical pipe with the flow traveling upward.

In order to quantify the performance of the developed algorithms in measurement of component volume fraction, a volumetric accuracy measure knows as Gas Volume Fraction Error (GVFE) defined using eqn. (8) is used.

$$
G V F E=\left|\alpha_{r e c}-\alpha_{r e f}\right| .
$$

where $\alpha_{\text {rec }}$ is the processed gas volume fraction on the basis of either the sampler or quantizer or thresholder, and $\alpha_{\text {rec }}$ is the reference gas volume fraction. The best results from the system should have minimum gas volume fraction error (GVFE).

$$
\alpha=\frac{\sum_{i=1}^{N} A_{i} \cdot g_{i}}{A_{\text {pipe }}}
$$

where $A_{i}$ is the area of pixel element $i, A_{\text {pipe }}$ is total area inside the pipe, $g_{i}$ is the grey level value for pixel $i$, and $N$ total number of pixel elements. 


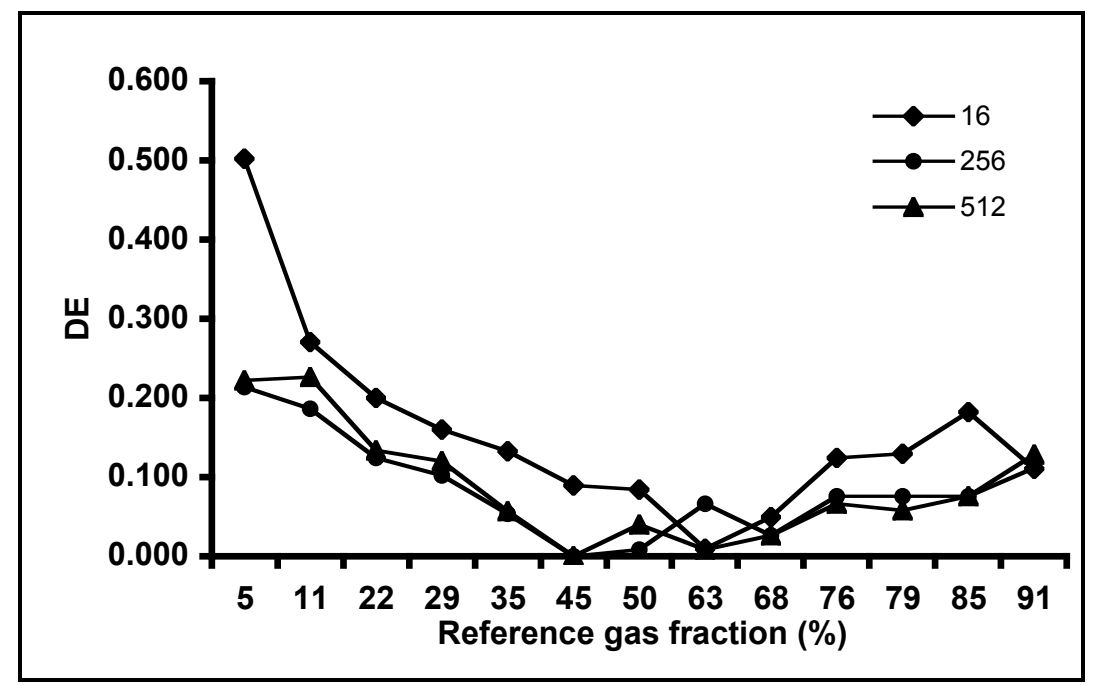

Figure 3: Performance of the KAP_SG entropic thresholding algorithm for different quantizer resolutions using computer simulated annular flow regime data.

\section{RESULTS AND DISCUSSION \\ Simulated static measurements}

Various simulated data covering the full component fraction range were processed using the quantizer and thresholder subsystems. The obtained tomograms were compared with reference tomograms to quantify the similarity between them using DE. The graphs of DE against reference volume fraction ranges were plotted to indicate their relative overall performances over the full component fraction range. Figure 3 shows the performance of the KAP SG entropic thresholding algorithm for different quantizer resolutions using computer simulated annular flow regime data. It can be seen that the performance of KAP SG thresholding algorithm improves with increasing quantizer resolution for simulated annular flow regime data tested. These simulated results suggest that higher resolutions are optimal for annular flow regimes. Further, the advantage of using 512 over the 256 quantizer resolution with the algorithm is marginal and has additional computational cost. On examining the performance of the counterpart thresholding algorithm, KAP SL using the same set of simulated data (figure 4), it can be seen that its performance on simulated annular flow data improves with increasing quantizer resolution, particularly in the higher gas concentration ranges. However, in lower gas concentration ranges, the advantage of higher resolution quantizer is not clear since both higher and lower resolutions almost show similar performance. Similarly, the advantage of using 512 over the 256 quantizer resolution with this algorithm is marginal. Theoretically, the 512 quantizer resolution is expected to give better thresholding performance than the 256 resolution since it would have significantly reduced the errors contributed by the quantization process. 


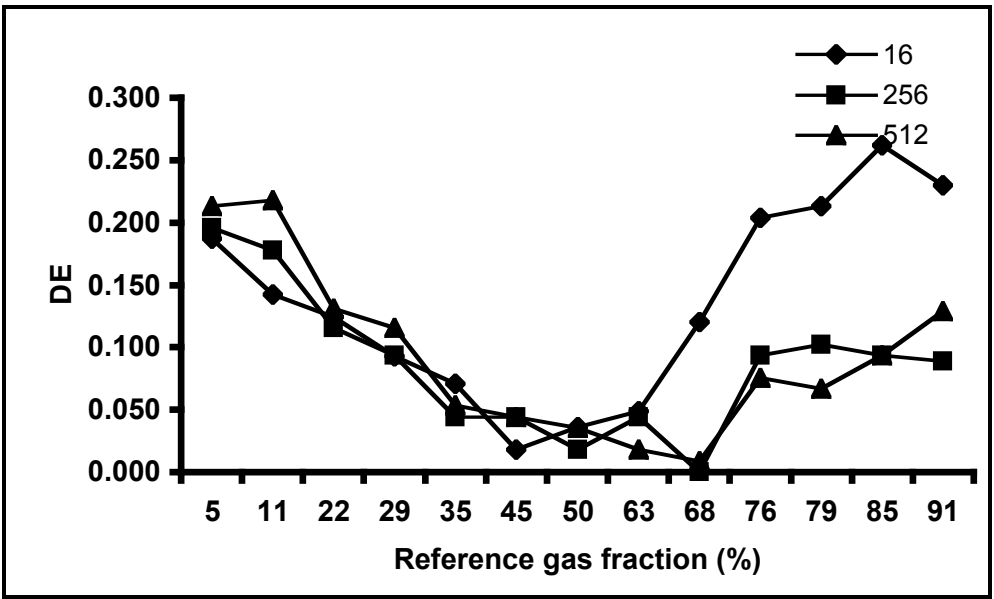

Figure 4: $\quad$ Performance of the KAP_SL entropic thresholding algorithm for different quantizer resolutions using computer simulated annular flow regime data.

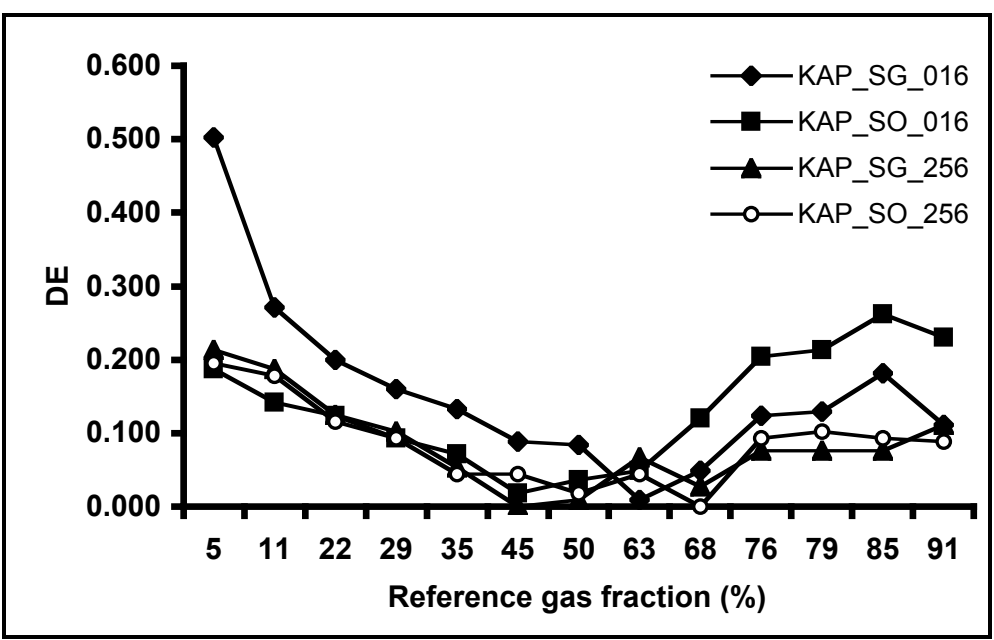

Figure 5: Performance comparison results between KAP_SG and KAP_SL entropic thresholding algorithms for different quantizer resolutions using computer simulated annular flow regime data.

Comparing the performance of the two thresholding algorithms using the same set of simulated data, KAP_SG and KAP_SL over the full component fraction range, it can be seen from figure 5 that their difference in performance is significantly larger in lower resolutions than in higher resolutions. For the sake of clarity, the 512 quantizer resolution results have been omitted in figure 5. From the same graphs, it can be seen that higher resolution minimizes the difference in performance between the two algorithms. This reduces the dependency of the thresholding algorithm on the nature of the object of interest searched, which in turn reduces the complexity of designing the thresholder. In other words, the properties of the 
thresholding algorithms will be less influenced by the nature of the selected searched object of interest during thresholding process. This can be seen in the performances of KAP SG and KAP SL for 256 quantizer resolution which are not very far apart.

\section{On-line dynamic measurements}

Various online data covering the full component fraction range were processed using the quantizer and thresholder subsystems. The obtained tomograms were compared with reference tomograms to quantify the similarity between them using GVFE. The graphs of GVFE against reference volume fraction ranges were plotted to indicate their relative overall performances. Figure 6 show the performance of the KAP SG entropic thresholding algorithm for different quantizer resolutions using on-line dynamic annular flow regime data. It can be seen that the performance of the KAP_SG thresholding algorithm for on-line measurement data partially agrees with the previous observations using the same algorithm for computer simulated data. There is improvement in the lower gas concentrations with increasing resolution but the situation is reversed in the higher gas concentrations where thresholding algorithm performance improves with decreasing resolution. This is unexpected because theoretically higher quantizer resolutions are supposed to perform better. However, overall results over the full component fraction range still suggest that higher resolution quantizer is optimal for annular flow percentage wise. The advantage of using higher quantizer resolution is distinctive in this case compared to simulated data performance (see figure 3), but still the advantage of using 512 over 256 quantizer resolution is marginal and with significant additional computational cost.

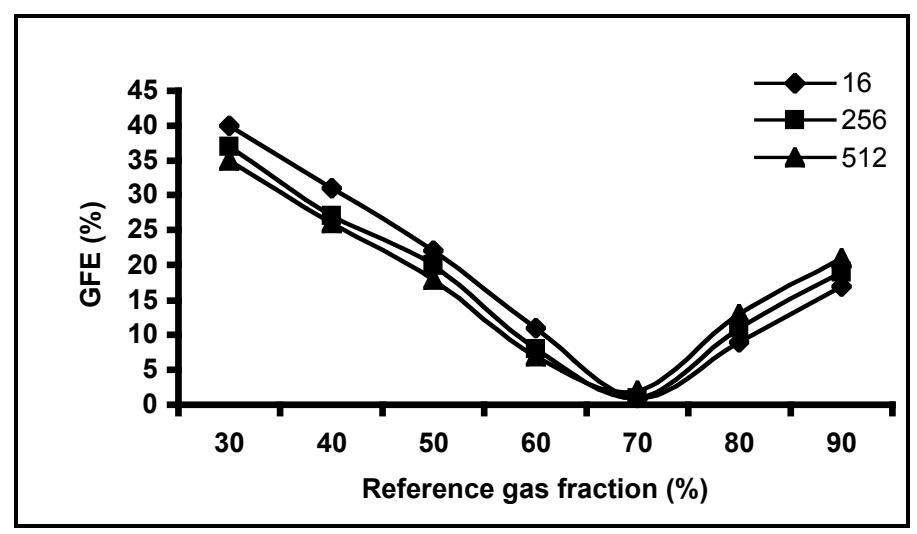

Figure 6: $\quad$ Performance of the KAP_SG entropic thresholding algorithm for different quantizer resolutions using on-line dynamic annular flow regime data.

Figure 7 show the performance of the KAP SL entropic thresholding algorithm for the same on-line set of data. From the graph it can be seen that on-line data clearly show that the performance of the KAP_SL thresholding algorithm for annular flow regime improve with decreasing quantizer resolution in lower gas concentration ranges, and vice versa in higher gas concentration ranges. The fuzzy distinction in the lower gas concentration ranges which was observed using simulated data (see figure 4) has been clarified. The superiority of KAP_SL over KAP_SG in the lower gas concentration ranges suggest that the choice of the object to be searched during thresholding process, 
gas or liquid for this case, cannot be ignored when lower gas concentration range data is concerned. Therefore it can be said that the choice of the object to be searched influences the performance of the entropic thresholding algorithm. Further, these results suggest that the assumption that by significantly reducing the distortions introduced by quantization process in the tomogram data will help improve the performance of thresholding algorithms is not directly applicable to KAP_SG and KAP_SL thresholding algorithms.

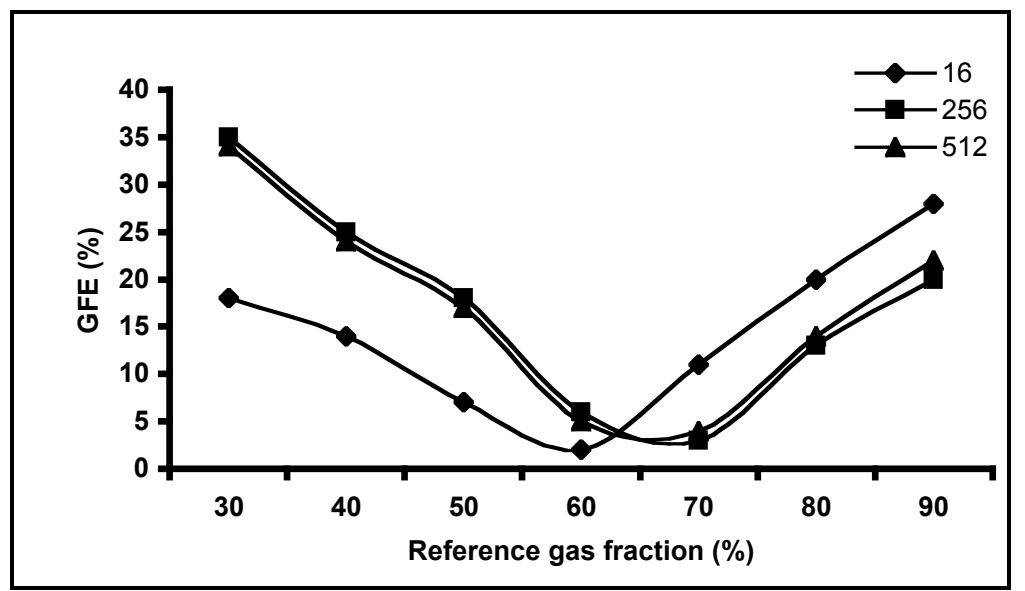

Figure 7: Performance of the KAP SL entropic thresholding algorithm for different quantizer resolutions using on-line dynamic annular flow regime data.

Similar observations with a few exceptions were made when the performance of the two thresholding algorithms KAP_SG and KAP_SL were compared using on-line data (see figure 8), as in the case of using simulated data. Higher resolutions significantly reduce the difference in performance between the two thresholding algorithms, although the performance of the KAP_SL algorithm is degraded in the lower gas concentration ranges. Therefore, it can be said that the dependency of the thresholding algorithm on the choice of the object to be searched during thresholding process can be minimized by using higher quantizer resolutions. The advantage of this situation is that entropic thresholding algorithms properties can be generalized regardless of the searched object used in the thresholding process. Also from the same graphs KAP_SG (256) and KAP_SL (16) are complementing each other over the full component fraction range. A data fusion algorithm, which picks the best of each algorithm, can be formed to improve the overall performance of the thresholding process over the full component fraction range. 


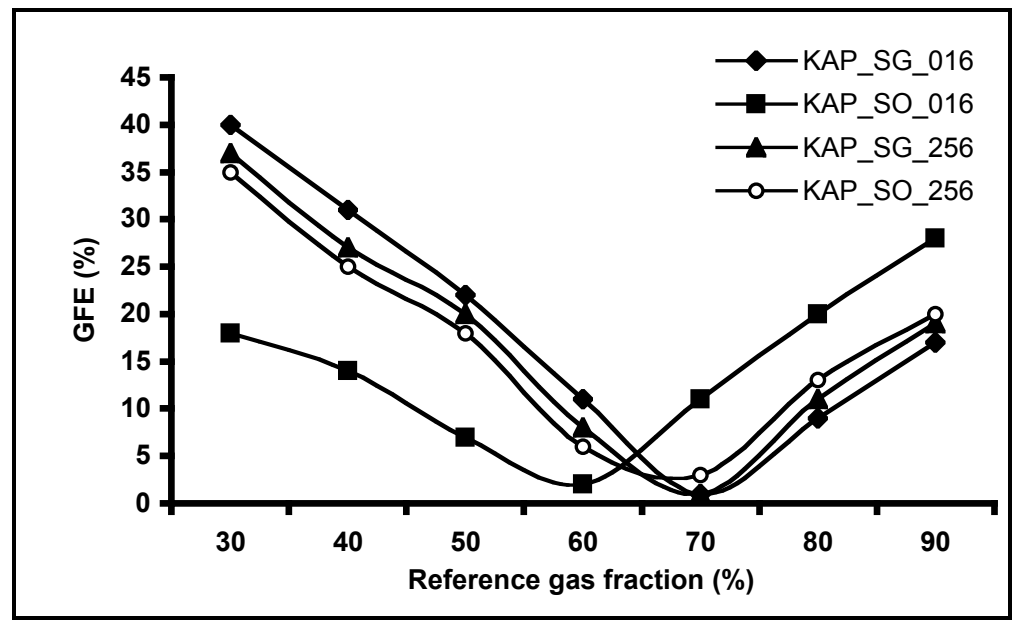

Figure8: Performance comparison results between KAP_SG and KAP_SL entropic thresholding algorithm for different quantizer resolutions using on-line dynamic annular flow regime data.

Both thresholding algorithms seem to be more accurate in the middle gas fraction ranges, creating a valley like pattern in all graphs over the full gas fraction range, regardless of the resolution used. This is one of the characteristics of the entropic thresholding technique used. The technique tends to bias toward splitting the grey level histograms into equal halves (Kapur, 1989). However, improvement in higher gas concentration ranges can be done by incorporating the truncation mechanism of Xie et al. (1992) as already demonstrated elsewhere (Mwambela and Johansen, 2001). It is difficult to minimize distortions in lower gas concentration ranges because of the following reason. The annular flow regime data used in the analysis, both simulated and on-line, are generated in such away that the smaller the gas concentration the smaller the air bubble at the center of the pipe. The problem with this is that the ECT sensory unit has poor sensitivity in the middle of the pipe cross section, hence small bubble in the middle of the pipe are poorly detected. Therefore the poor performance of the algorithms in the lower ranges can also be attributed to the ECT sensory unit whose sensitivity in the middle of the pipe is known to be poor.

From the analysis of the results for both simulated and on-line data, entropic thresholding algorithms applicability in multiphase flows component volume fraction measurement can be conceived as two types depending on the component distribution. The KAP_SG (256) thresholding algorithm is suitable for flow regimes with high gas concentrations whereas KAP_SL (16) is for suitable for flow regimes with lower gas concentrations. Since these two algorithms are also complementing each other over the full component fraction range, a data fusion mechanism can be used to combine the best of each algorithm to give an overall better performance.

The assumption that higher quantizer resolution, which have the effect of significantly minimizing distortions contributed by the quantization process, will help improve the performance of entropic thresholding algorithms seems to be true only for KAP SG algorithm. There is a significant deviation for the KAP_SL 
algorithm in the lower range of gas concentrations, where KAP SL (16) outperform those algorithms with higher resolution. Further experiment is required using other algorithms to see whether this is a property of the entropic thresholding or the system and data used. Although higher resolution are optimal for annular flows and KAP_SG algorithm, the performance gained by using the 512 over 256 quantizer resolution is marginal compared to the computational cost added. A compromising quantizer resolution between accuracy and computational cost for this case therefore is 256.

Although quantization process introduces irreversible distortions in the data passing through it, significant distortions are introduced by the LBP reconstruction algorithm itself. Therefore, further work is needed to improve the accuracy of the LBP algorithm before more complex quantization process can be considered for evaluation.

The nature of the object to be searched by the thresholder influences the accuracy of the algorithms. When gas or liquid is the object of interest, the performances will vary over the full component fraction range. The variations are significant when lower resolutions are used as demonstrated in this work where for instance KAP SG (16) is significantly different from KĀP_SL (16). This influence of the object to be searched on the entropic thresholding algorithms can be minimized using higher resolutions. In this way we can generalize the properties of the entropic thresholding in relation to the measurement data used with minimum deviations.

Although higher resolutions are optimal for annular flow regimes, the final use of the entropic thresholding algorithm has to consider other factors as well. Complementarities of the algorithms, speed of reconstruction, accuracy and the nature of object to be searched can be taken into considerations when necessary.

\section{CONCLUSION}

Optimal quantizer resolution depends on the nature of the component distribution in the multi phase flow. Global entropic thresholding algorithms perform better for annular flow regimes using higher quantizer resolutions and KAP_SG thresholding algorithm in higher gas concentration ranges. However, in lower gas concentration ranges better performance is achieved using lower quantizer resolutions and KAP SL thresholding algorithm. Higher quantizer resolutions minimize the dependency of the entropic thresholding algorithm on the nature of the object to be searched during threshing process. In general it can be said that 256 is the optimal quantizer resolution which give better compromise between reconstruction speed and measurement accuracy.

\section{ACKNOWLEDGEMENT}

The author wishes to thank P. Msaki and anonymous reviewers for their useful comments. This work has been supported by SIDA/SAREC through the core support program.

\section{REFERENCES}

Galbiati LJ 1990. Machine vision and digital image processing fundamentals. Prentice-Hall, ISBN 0-13-541988-3

Gray RM and Neuhoff D. L, 1998. Quantization. IEEE Trans. Info. Theory, 44(6): 2325 - 2383.

Isaksen Ø 1994. A novel approach to reconstruction of process tomography data. Ph.D. Thesis, University of Bergen, Norway.

Isaksen Ø, 1996. A review of reconstruction techniques for capacitance tomography. Meas. Sci. Technol. 7:325 - 337.

Kapur JN 1989. Maximum entropy models in science and engineering. New Delhi: Wiley

Kapur JN, Sahoo PK and Wong ACK 1985. A new method for grey level picture thresholding using the entropy of the histogram, Com. Vision, Graphics, and Image Processing 29, pp. 273-285. 
Midttveit $\varnothing$ and Aspelund A 1997. CMR Multiphase test facility: System description, CMR-96-F10025.

Mwambela AJ 1999. Reconstruction of capacitance tomography data using entropic thresholding techniques, Ph.D. Thesis, University of Dar es Salaam, TANZANIA.

Mwambela AJ and Johansen GA 2001. Multiphase flow component volume fraction measurement: experimental evaluation of entropic thresholding methods using electrical capacitance tomography system, Meas. Sci. Technol. 12:1092-1101.

Mwambela AJ, Isaksen Ø and Johansen GA 1997. The use of entropic thresholding methods in reconstruction of capacitance tomography data, J. Chemical Engineering Science 52(13):2149-2159.

Shannon CE and Weaver W 1946. The mathematical theory of communication. University of Illinois Press, Urbana.
Waritso W and Fan LS 2001. Neural network based multi-criterion optimization image reconstruction technique for imaging two and three phases flow systems using electrical capacitance tomography, Meas. Sci. Technol. 12:2198-2210.

Xie CG, Huang SM, Hoyle BS, Thorn R, Lenn C and Beck MS 1992. Electrical capacitance tomography for flow imaging: System model for development of reconstruction algorithms and design of primary sensors, IEE Proc. 139:8698.

Yang W 1996. Hardware design of electrical capacitance tomography system. Meas. Sci. Technol. 7:225-232.

Yang W 2001. Further developments in an ac-based capacitance tomography system. Rev. Sci. Instrum. Vol 72(10): pp 39023907. 